\title{
ESTABLISHMENT OF THE CENTER FOR ADVANCED SEPARATION TECHNOLOGIES
}

\author{
ANNUAL TECHNICAL PROGRESS REPORT \\ Report Period \\ October 1, 2004 to September 30, 2005
}

Compiled by

Christopher E. Hull

Issued January 16, 2005

DOE Award Number:

DE-FC26-01NT41091

Center for Advanced Separation Technologies

Virginia Polytechnic Institute \& State University

Blacksburg, Virginia 24061-0258

and

National Research Center for Coal and Energy

West Virginia University

Morgantown, WV 26506-6064 


\section{DISCLAIMER}

This report was prepared as an account of work sponsored by an agency of the United States Government. Neither the United States Government nor any agency thereof, nor any of their employees, makes any warranty, express or implied, or assumes any legal liability or responsibility for the accuracy, completeness, or usefulness of any information, apparatus, product, or process disclosed, or represents that its use would not infringe privately owned rights. Reference herein to any specific commercial product, process, or service by trade name, trademark, manufacture, or otherwise does not necessarily constitute or imply its endorsement, recommendation, or favoring by the United States Government or any agency thereof. The views and opinions of authors expressed herein do not necessarily state or reflect those of the United States Government or any agency thereof. 


\begin{abstract}
This Technical Progress Report describes progress made on the eight sub-projects awarded in the first year and the five projects awarded in the second year of Cooperative Agreement DE-FC26-01NT41091: Establishment of the Center for Advanced Separation Technologies. This work is summarized in the body of the main report: the individual subproject Technical Progress Reports are attached as Appendices.
\end{abstract}

Note: SI is an abbreviation for "Le Systeme International d’Unites." 


\section{TABLE OF CONTENTS}

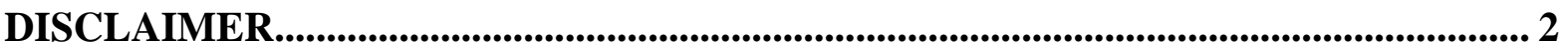

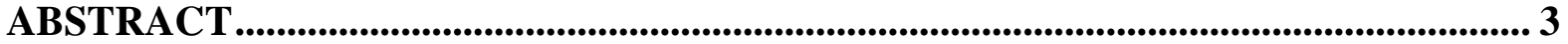

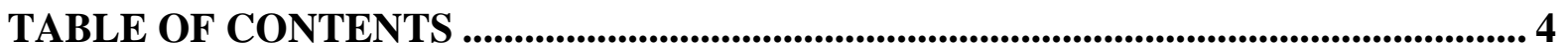

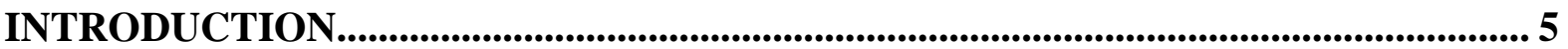

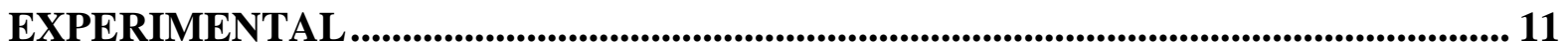

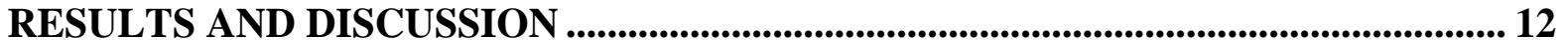

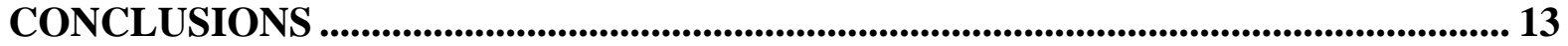

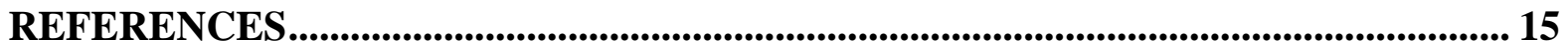

APPENDIX A: EVALUATION OF COAL CLEANING EFFICIENCY USING

TRANSPONDER-BASED DENSITY TRACERS................................................... 16 


\section{INTRODUCTION}

The U.S. is the largest producer of mining products in the world. In 1999, U.S. mining operations produced $\$ 66.7$ billion worth of raw materials that contributed a total of $\$ 533$ billion to the nation's wealth. Despite these contributions, the mining industry has not been well supported with research and development funds as compared to mining industries in other countries. To overcome this problem, the Center for Advanced Separation Technologies (CAST) was established by Virginia Tech and West Virginia University to develop technologies that can be used by the U.S. mining industry to create new products, reduce production costs, and meet environmental regulations. This endeavor is supported through U.S. DOE Cooperative Agreement No. DE-FC26-01NT41091: Establishment of the Center for Advance Separation Technologies.

Much of the research to be carried out at CAST will be longer-term, high-risk, basic research, and will be carried out in four broad areas:
a) Solid-solid separation
b) Solid-liquid separation
c) Chemical/Biological extraction, and
d) Sensor and control development.

Distribution of funds has been handled via competitive solicitation of research proposals through Site Coordinators at the two universities. Two RFP's have been issued to date. The first of these, referred to as the CAST I - Round 1 RFP, was issued on August 17, 2001 and closed October 15, 2001. A total of 12 proposals were received in response to this first RFP. These were first reviewed and ranked by a group of technical reviewers (selected primarily from industry). Based on these reviews, and an assessment of overall program requirements, the CAST Technical Committee made an initial selection/ranking of proposals and forwarded these proposals to the DOE/NETL Project Officer for final review and approval. This process took some 6 months to complete but 8 projects were in place at the constituent universities ( 5 at Virginia Tech and three at West Virginia University) by May 17, 2002.

This was followed, on June 1, 2002 with the CAST I - Round 2 RFP, which closed on July 19, 2002. A total of 12 proposals were received in response to this second RFP five were selected for funding. These were in place at the constituent universities (2 at Virginia Tech and 3 at West Virginia University) by March 1, 2003.

All thirteen of these projects are listed below by category, along with brief abstracts of their aims and objectives. 


\section{a) Solid-Solid Separation}

\section{Improving Coarse Particle Flotation}

Principal Investigators: R.-H. Yoon and G.H. Luttrell, (Virginia Tech).

Period of Performance: May 17, 2002-May 31, 2006.

The recovery of coarse particles by flotation is a common problem in the coal and minerals processing industry. The objective of this project is to develop methods of extending the upper particle size limit for flotation. The availability of such a technology will improve processing efficiency and greatly simplify the plant flowsheet. Two different complementary approaches will be used to achieve the project objective. One is to use novel flotation reagents that will help increase the attachment force between air bubbles and coarse particles, and the other is to develop new flotation machines specifically designed to promote coarse particle flotation.

\section{Separation of Small Particles due to Density Differences in a CFB Riser System} Principal Investigators: E. Johnson and B. Kang (West Virginia University). Period of Performance: May 17, 2002-May 31, 2006.

The efficiency of separating small (less than 250 micrometers in diameter) dense particles from small light particles in the internal recirculation flow within a CFB riser is being investigated. Currently, there are many systems for separating fine particles. However, these systems lack the efficiency and/or the economics to be commercially viable in many situations. This research is directed towards removing pyrite from coal fines at a coal cleaning plant. Also, of concern will be the removal of radioactive particles from the desert sand at the Nevada Test Sites.

\section{Modeling of Flotation From First Principles}

Principal Investigator: R.-H. Yoon (Virginia Tech).

Period of Performance: May 17, 2002-May 31, 2006.

Flotation is widely regarded as the best available technology for separating fine particles. The present form of the technology was invented nearly 100 years ago, yet there is still no reliable model for predicting flotation rate from first principles. It is the purpose of the proposed work to develop a flotation model that can predict flotation performance from both surface chemistry and hydrodynamic parameters.

\section{Studies of Froth Stability and Model Development}

Principal Investigator: R.-H. Yoon (Virginia Tech).

Period of Performance: May 17, 2002-May 31, 2006.

Froth plays an important role in flotation. It determines the final grade of the product and the maximum carrying capacity (or throughput) of a flotation machine. Also, many operators use stronger frothers to produce smaller air bubbles and, hence, higher recovery and throughput. Despite its importance, little is known of the fundamentals of foam and froth stability. It is, therefore, proposed to study the various factors affecting the stability 
of flotation froth. This will be accomplished by using a thin film balance technique and by monitoring the stability of froth in a bubble column using a video camera. The results will be used for developing a froth model and also for developing effective defoamers.

\section{Direct Measurement of Forces in Flotation Systems}

Principal Investigators: W.A. Ducker and R.-H. Yoon (Virginia Tech).

Period of Performance: May 17, 2002-May 31, 2006.

The objective of this project is to directly measure the force as a function of separation between a particle and a bubble in aqueous solution. This force controls the attachment and detachment of particles to bubbles, which is an essential step in determining the efficiency of the separation of mineral particles in a flotation cell. We are fabricating a device specially designed for these measurements. The device will use a force-detection method from an Atomic Force Microscope and a separation-detection method from a Surface Forces Apparatus. The key advances are to explicitly measure both the separation between the particle and the bubble and the shape of the bubble at all times. Without this separation and shape data, it is not possible to compare the measured forces to theoretical estimates or to the practice of flotation. After fabrication of the device, measurements of the forces acting on hydrophilic, hydrophobic, and charged particles in aqueous solutions of surfactant molecules will be obtained.

\section{Novel Surfactants as Collectors for Froth Flotation}

Researcher: Richard D. Gandour (Virginia Tech)

Period of Performance: March 1, 2003-May 31, 2006.

Our goals are to synthesize novel, inexpensive surfactants that outperform current collectors in mineral flotation. These surfactants will selectively bind to particles and create a hydrophobic coating on a particle to enable selective separation by froth flotation. We plan to synthesize surfactants that have very hydrophobic, long chains, yet have sufficient water solubility for mineral processing and coal-fines processing. These amphiphiles, which we call "hydra surfactants," will have one tail and three identical functional groups on the head. These novel molecules will be useful for many different separations in mineral processing, but initially will be tested on separation of discoloring impurities (i.e., anatase (TiO2) and iron oxides) from clay.A strategy to make these surfactants from environmentally friendly vegetable oils is outlined.

\section{Column Flotation of Fine Dolomitic Phosphate by Selective Fatty Acids}

Researcher: F. F. Peng (West Virginia University)

Period of Performance: March 1, 2003-May 31, 2006.

Among the detrimental impurities in phosphate ores, dolomite is considered the most troublesome for down stream operations. Dolomite may cause higher consumption of sulfuric acid, reduce filtration rates and lower $\mathrm{P}_{2} \mathrm{O}_{5}$ content in fertilizer manufacturing processes. Difficulties in separating dolomite from phosphate arise from the fact that most of the dolomite is disseminated in the phosphate mineral. Both are oxide type minerals and have the same calcium cationic component. They exhibit similar 
electrokinetics, adsorption and desorption behavior in physical separation process such as flotation. Several processing schemes have been developed to handle this problem, but none are satisfactory due to high $\mathrm{MgO}$ content and/or low overall $\mathrm{P}_{2} \mathrm{O}_{5}$ recovery in the final phosphate concentrate. In this proposed research project, samples from Florida dolomitic phosphate minerals will be tested in an open flotation column. New selective fatty acid collectors for dolomite particles and appropriate mixtures of phosphoric acid/sulfuric acid depressant for phosphate particles will be selected and used. The aim is to achieve a phosphate concentrate containing as high as $30 \% \mathrm{P}_{2} \mathrm{O}_{5}$ and less than $1.0 \%$ MgO contents at high $\mathrm{P}_{2} \mathrm{O}_{5}$ recovery. Liberation analysis of dolomitic phosphate pebble will be conducted. The selectivity and frothability of the new selective fatty acid collectors will also be characterized by determining dolomitic phosphate flotation. Success in this research project will enable the mineral industry to increase its phosphate reserve base, by recovering phosphate from formerly unusable phosphatic minerals and low grade phosphate reserves.

\section{b) Solid-Liquid Separations}

\section{Development of a Novel Fine Particle Centrifuge}

Researchers: G.H. Luttrell and R-H. Yoon (Virginia Tech)

Period of Performance: March 1, 2003-May 31, 2006.

The solid-solid separation processes employed by modern coal preparation plants require large amounts of process water. After cleaning, the unwanted water must be removed from the surfaces of the particles using mechanical dewatering equipment. Coarse particles can be readily dewatered using simple screening systems, while finer particles require more complicated unit operations such as centrifuges and filters. Unfortunately, the processes used to dewater fine particles are inherently inefficient and expensive to operate and maintain. To overcome these problems, a novel centrifugal filter has been developed by researchers at Virginia Tech. Preliminary test data suggest that this new technology can reduce the moisture content of fine coal products by approximately $30-50 \%$ compared to existing dewatering processes. The objective of this project will be to construct a continuous prototype unit and to conduct a detailed experimental investigation of this new technology.

\section{New Strategies for Dewatering of Coals}

Researchers: M. Seehra, A. Manivannan \& M. Bachlechner (West Virginia University)

Period of Performance: March 1, 2003-May 31, 2006.

A two-year innovative research program on the combined experimental-modeling studies of coal-water interactions and coal dewatering is proposed. The experimental program will first focus on determining the relative amounts of "free" and "bound" water in wet coals using analytical techniques of thermogravimetric analysis, heat capacity measurements and $1 \mathrm{H}$ nuclear magnetic resonance. Laboratory scale dewatering experiments will then be carried out by vacuum filtration and centrifugation, combined with pulsed heating at microwave or IR $(3300 \mathrm{~cm}-\mathrm{l})$ frequencies to liberate the "bound" water. These investigations will be complimented with modeling studies of the coal-water 
interactions and the effect of centrifugation on dewatering from surface-bound water and coal pores. This integrated experimental-modeling approach is an important component of the proposed research since the results from the molecular-dynamics simulations could provide unique technological insights that will increase the efficiency of dewatering processes. Finally, scale-up of the resulting successful approaches for coal dewatering will be proposed at the conclusion of the two-year program.

\section{c) Chemical/Biological Extraction}

\section{Novel Bioleaching Technology Assisted by Electrolytic Processes}

Principal Investigators: E. Cho and R. Yang (West Virginia University).

Period of Performance: May 17, 2002-May 31, 2006.

Bioleaching of sulfide minerals using bacteria Thiobacillus ferrooxidans has been identified as a promising method for heap or dump leaching of low grade mixed sulfide ores containing pyrite $\left(\mathrm{FeS}_{2}\right)$, chalcopyrite $\left(\mathrm{CuFeS}_{2}\right)$, and/or sphalerite ( $\left.\mathrm{ZnS}\right)$. However, the major problem with bioleaching is that the rates are too slow for wide commercial utilization. The approach being used in this project focuses on the growth of bacteria under the influence of an applied direct potential and on the utilization of the bacteria to leach sulfide minerals. The objective is to identify the optimum conditions for the bioand electro- leaching reactions in order to maximize overall leaching rates of the sulfide minerals.

\section{Coal Desulfurization Using Hypochlorite and Cupric Ion as a Catalyst - Feasibility Study}

Researchers: E. H. Cho \& R. Y. K. Yang (West Virginia University)

Period of Performance: March 1, 2003-May 31, 2006.

The utilization of coal as a feedstock to produce carbon materials is very much hampered by the presence of its organic sulfur content. However, the organic sulfur is very difficult to remove. It is generally accepted that only two methods have capability to remove the organic sulfur rather effectively; one is with a melt of sodium hydroxide/potassium hydroxide at high temperatures (Gravimelt Process) and the other is with chlorine chemicals. However, the Gravimelt Process contaminates the coal structure, and its decontamination and the regeneration of the reagents may be insurmountable problems in terms of economic feasibility. Chlorine chemicals also chlorinate the coal structure and the removal of organic sulfur is not high enough to produce a premium coal or feedstock. It is proposed that organic sulfur be leached with hypochlorite using cupric amine as a catalyst. Hypochlorite is the predominant chlorine derivative at high $\mathrm{pH}$ 's. The catalysis of cupric amine was found during 1990s in leaching of gold with hypochlorite and other oxidants. We will conduct two kinds of consecutive experiments. In the first, coal will be leached in a reactor with a solution containing hypochlorite and cupric amine. In the second, the leached coal will be hydrolyzed in hot sodium carbonate solution, which is believed to cleave the organic sulfur bond from coal and thus further reduce the sulfur content. Leached coal and hydrolyzed coal samples will be analyzed particularly for organic sulfur and chloride content. The data will be analyzed to 
determine the desulfurization potential through the two consecutive experiments and also the chlorination/dechlorination of the coal matrix through each of the two experiments.

\section{d) Sensor and Control Development}

\section{Development of Electrochemical Sensor for On-Site Monitoring of Heavy Metal Ions in Coal Processing And Utilization}

Principal Investigators: A. Manivannan and M. Seehra (West Virginia University). Period of Performance: May 17, 2002-May 31, 2006.

The aim of this research program is to develop a novel electrochemical sensor based on a boron-doped diamond (BDD) electrode for monitoring/controlling heavy metal ions such as $\mathrm{Hg}, \mathrm{Zn}, \mathrm{Cu}, \mathrm{Pb}, \mathrm{As}, \mathrm{Cd}$, and $\mathrm{Fe}$ encountered in the processing and utilization of coals. This research is based on earlier testwork in which ppb levels of $\mathrm{Pb}$ and Hg were detected in laboratory prepared solutions. BDD electrodes are superior to other commonly used electrodes (such as glassy carbon) in terms of their ruggedness, chemical stability, wide potential window and lower background current. These advantages are important for the simultaneous detection of a number of elements in solution.

\section{Evaluation of Coal Cleaning Efficiency Using Transponder-Based Density Tracers} Principal Investigators: G.H. Luttrell, R.-H. Yoon and C.J. Wood (Virginia Tech). Period of Performance: May 17, 2002-May 31, 2006.

Density tracers are one of the most powerful diagnostic tools for evaluating the performance of heavy media circuits in coal preparation plants. The objective of this project is to develop a new generation of tracers that can be automatically tracked using recently developed transponder technology. This development also makes it possible for efficiency tests to be performed very rapidly by a single person in an extremely cost-efficient manner. The project will be carried out in two phases. In the first phase of work, the hardware and software required to develop a prototype transponder-based system will be procured and evaluated using a simulated circuit at the Virginia Tech coal laboratory. In the second phase, the monitoring system will be relocated to an industrial coal preparation plant for field-testing. 


\section{EXPERIMENTAL}

The CAST initiative is comprised of a diverse group of subprojects, most of which are multistage, task-oriented developmental projects that cannot be conveniently categorized by the traditional reporting criteria required by the DOE Uniform Reporting Requirements. For example, several of the projects have required the construction of unique test equipment, others the generation of simulation models, etc., as preliminary tasks in the overall execution of the project. As such, they are more appropriately described and discussed as "Project Tasks" within the context of the individual Technical Progress Reports. These reports are attached to this document as Appendices and should be referred to for this information. 


\section{RESULTS AND DISCUSSION}

The CAST initiative is comprised of a diverse group of subprojects, most of which are multistage, task-oriented developmental projects that cannot be conveniently categorized by the traditional reporting criteria required by the DOE Uniform Reporting Requirements. For example, several of the projects have required the construction of unique test equipment, others the generation of simulation models, etc., as preliminary tasks in the overall execution of the project. As such, the presentation of results is more appropriately described and discussed within the context of the individual Technical Progress Reports. These reports are attached to this document as Appendices and should be referred to for this information. 


\section{CONCLUSIONS}

This aggregated Technical Progress Report covers the third year of operation of Cooperative Agreement DE-FC26-01NT41091: Establishment of the Center for Advanced Separation Technologies. A total of thirteen (13) sub-projects are currently funded: twelvemonthly Progress Reports (covering the period October 1, 2003-September, 2004) have been generated for 10 of these projects which are still in progress. These are attached to this document as Appendices. Three (3) projects have been completed and final reports will be issued separately. A brief summary of progress during the year, along with plans for the future, is given below for each of the sub-projects.

\section{a) Solid-Solid Separation}

1. VA003 - Improving Coarse Particle Flotation

Principal Investigators: R.-H. Yoon and G.H. Luttrell (Virginia Tech)

Period of Performance: May 17, 2002-May 31, 2006

The project is completed and a final report is to be issued.

2. WV002 - Separation of Small Particles due to Density Differences in a CFB Riser System

Principal Investigators: E. Johnson and B. Kang (West Virginia University)

Period of Performance: May 17, 2002-May 31, 2006

The project is completed and a final report is to be issued.

\section{VA001 - Modeling of Flotation From First Principles}

Principal Investigator: R.-H. Yoon (Virginia Tech)

Period of Performance: May 17, 2002-May 31, 2006

The project is completed and a final report is to be issued.

\section{VA002 - Studies of Froth Stability and Model Development}

Principal Investigator: R.-H. Yoon (Virginia Tech)

Period of Performance: May 17, 2002-May 31, 2006

The project is completed and a final report is to be issued.

5. VA004 - Direct Measurement of Forces in Flotation Systems

Principal Investigators: W.A. Ducker and R.-H. Yoon (Virginia Tech)

Period of Performance: May 17, 2002-May 31, 2006

The project is completed and a final report is to be issued. 


\section{VA007 - Novel Surfactants as Collectors for Froth Flotation}

Researcher: Richard D. Gandour (Virginia Tech)

Period of Performance: March 1, 2003-May 31, 2006

The project is completed and a final report is to be issued.

7. WV006 - Column Flotation of Fine Dolomitic Phosphate by Selective Fatty Acids Researcher: F. F. Peng (West Virginia University)

Period of Performance: March 1, 2003-May 31, 2006

The project is completed and a final report is to be issued.

\section{e) Solid-Liquid Separations}

8. VA006 - Development of a Novel Fine Particle Centrifuge Researchers: G.H. Luttrell and R-H. Yoon (Virginia Tech) Period of Performance: March 1, 2003-May 31, 2006

The project is completed and a final report is to be issued.

9. WV004 - New Strategies for Dewatering of Coals

Researchers: M. Seehra, A. Manivannan \& M. Bachlechner (West Virginia University)

Period of Performance: March 1, 2003-May 31, 2006

The project is completed and a final report is to be issued.

\section{b) Chemical/Biological Extraction}

10. WV001 - Novel Bioleaching Technology Assisted by Electrolytic Processes Principal Investigators: E. Cho and R. Yang Period of Performance: May 17, 2002-May 31, 2006

The project is completed and a final report is to be issued.

11. WV005 - Coal Desulfurization Using Hypochlorite and Cupric Ion as a Catalyst Feasibility Study

Researchers: E. H. Cho \& R. Y. K. Yang (West Virginia University)

Period of Performance: March 1, 2003-May 31, 2006

The project is completed and a final report is to be issued.

c) Sensor and Control Development

12. WV003 - Development of Electrochemical Sensor for On-Site Monitoring of Heavy Metal Ions in Coal Processing And Utilization

Principal Investigators: A. Manivannan and M. Seehra (West Virginia University)

Period of Performance: May 17, 2002-May 31, 2006 (2-Year Project) 
The project is completed and a final report is to be issued.

\section{VA005 - Evaluation of Coal Cleaning Efficiency Using Transponder-Based Density Tracers (Appendix J) \\ Principal Investigators: G.H. Luttrell, R.-H. Yoon and C.J. Wood \\ Period of Performance: May 17, 2002-May 31, 2006}

Field tests were carried out to evaluate the capabilities of the proposed electronic detection system for counting density tracers. The test runs were performed by passing density tracers embedded with passive transponders through a heavy medium cyclone circuit at an industrial plant site. Several problems were encountered during the initial test run that resulted in poor detection levels for the electronic tracers. These problems, which included improper antenna heights and excessively rapid tracer introduction, were corrected by simple changes to the radio equipment and test protocols. After making these modifications, very accurate counts of the transponder-based tracers were achieved. In the final test run, $98.5 \%$ of the electronically tagged tracer blocks were detected by the automated counting system. This level of detection was superior to the $92.3 \%$ collection rate achieved using manual (hand) collection. The superior performance was particularly impressive considering that two employees were used per $6 \mathrm{ft}$ width of screen to ensure that very high manual collection rates were maintained. The manual collection rate is normally much lower in actual practice since (i) fewer personnel are normally available for collecting the density tracers and (ii) few plant sites are as ideally suited for block collection as the one employed for this test work.

Work conducted during the next reporting period will focus on further refinement of the user interfaces for the electronic density tracer system. In addition, a cost analysis will be performed to assess the overall cost of installing and operating the prototype system at an industrial plant site. Work will also begin on the preparation of a final technical report for this research project.

\section{REFERENCES}

References utilized by the individual sub-projects are reported in the relevant Technical Progress Report in the attached Appendices. 
Appendix A: Evaluation of Coal Cleaning Efficiency Using TransponderBased Density Tracers 


\section{TECHNICAL PROGRESS REPORT}

\begin{tabular}{|c|c|}
\hline Contract Title and Number: & Period of Performance: \\
\hline & \\
\hline Technologies (DE-F & Ending Date: \\
\hline
\end{tabular}

Sub-Recipient Project Title:

Evaluation Coal Cleaning Efficiency Using

Transponder-Based Density Tracers

Principal Investigators:

Gerald Luttrell and Roe-Hoan Yoon

Contact Address:

146 Holden Hall

Virginia Polytechnic Institute \& State University

Blacksburg, VA

Subcontractor Address:

No subcontracts issued.
Report Information:

Type: Annual

Number: 3

Period: $\quad$ 10/1/04-9/30/05

Date: $\quad$ 10/30/05

Code: VA005-R04

Contact Information:

Phone: (540)231-4508

Fax: (540)231-3948

E-Mail: $\quad$ cast@vt.edu

Subcontractor Information:

Phone:

Fax:

E-Mail:

\section{ABSTRACT}

Density tracers are one of the most powerful diagnostic tools for evaluating the performance of coal preparation plants. The objective of this project is to develop a new generation of density tracers that can be automatically tracked using RFID (radio frequency identification) technology. During previous reporting periods, controlled experiments were performed with static (stationary) transponders to evaluate the response of the RFID detection system to variables such as tracer-antenna orientation, detection distance, signal interference, reliability, etc. The hardware and software required to develop the transponderbased system were also procured and evaluated. This effort included the manufacture of density tracers that incorporate electronic transponder tags and the construction of stationary readers for identifying the counting the tagged tracers. Electronic interfaces and graphical software were also developed to convert the tracer counts into efficiency curves for use by plant personnel. The prototype system was tested and evaluated at the Virginia Tech coal laboratory using a simulated circuit. During the past reporting period, the monitoring system was relocated to an industrial coal preparation plant for field-testing. A regional coal company provided an industrial site for the testing of these new transponder-based density tracers. 


\section{INTRODUCTION}

\section{Background}

Density traces are plastic blocks (usually cubic) that incorporate high-density fillers to create artificial particles with known densities in the range of 1.20-2.60 SG. Density tracers offer a rapid and low-cost method for identifying problems with density-based processes that currently serve as the workhorse for the coal preparation industry. A typical plant test requires about an hour to complete as compared to several days (or even weeks) for traditional sampling and laboratory analysis. In addition, the tracer procedure is less costly and less hazardous to the environment compared to competitive float-sink tests that utilize toxic organic liquids. Unfortunately, the use of density tracers in industrial operations has been limited due to problems that occur during the retrieval step. The amount of labor required to manually collect the blocks can be excessive for large plants or complicated circuits. In addition, the data can sometimes be unreliable since tracers can be easily lost when buried in a thick bed of particles.

\section{Objective and Approach}

The objective of this project is to develop a new type of density tracer that can be automatically tracked using an electronic tagging system. The proposed system makes use of miniature tags (transponders) that are embedded within the tracer blocks when they are manufactured. These "passive" tags remain inactive until they become externally powered by a transmission signal from an antenna of a remote interrogator/reader. Once energized, the tags transmit a unique digital code back to the interrogator/reader that identifies the density and size of tracers reporting to either the clean coal or refuse products. The successful development of this technology will eliminate statistical counting errors associated with lost tracers and will make it possible for tests to be performed by a single individual. During the past reporting period, a Texas Instruments RFID system was tested to see if this low-cost, off-the-shelf technology could meet the strict requirements of the density tracer application.

\section{PROJECT TASKS}

\section{Task 6 - Software Development}

Considerable work was performed during current reporting period to develop the software required for the transponder-based density tracer system. This activity included the construction of a suitable user interface, programming of the computation algorithms, and the completion of required logic interfaces between the transponder system and computer. The user interface was designed to provide a direct graphical readout showing the process efficiency curve and pertinent performance indicators (e.g., SG cutpoint and Ep). This information can be directly used by plant operators to evaluate the on-line circuit performance and estimate coal losses. A Visual Basic program interfaced with the reader hardware was developed to capture and record the tracer identification numbers. Once the collection step was completed, the data was processed using Excel VBA and Excel SOLVER for reporting of the final results. A COM interface was also written in Visual Basic to control 
the acquisition of data from the antenna array. This program automatically cycles through the four antennas to record the location where each transponder was read. Proper tuning of the antenna array was found to be extremely important in achieving the optimum read range for the transponder-based tracers.

\section{Task 7 - Field Testing}

Final testing of the proposed system was conducted at an industrial coal preparation plant. The test site was selected so that the tagged density tracers could be easily recovered by plant personnel and reused in additional test runs. Because of the preliminary nature of this work, the plant trials were limited to testing of a relatively simple heavy medium cyclone circuit with a minimal number (only four) drain and rinse screens. This limitation was necessary because of the small number of reader antenna (only four) and associated hardware purchased using the modest project budget.

During testing, the four antennas were suspended above the drain and rinse screens using chains. The use of chain connectors allowed for easy installation, adjustment and removal of the antennas. Two antennas were required for each drain and rinse screen in order to fully cover the width of the six foot wide screens. In order to be sure of no interference between the antennas, one antenna was placed close to the end where the material comes onto the screen and one was positioned near the end where the material was discharged (Figure 1). For the end closest to the feed end of the screens, the chains holding the antennas were supported from C-clamps attached to the underside of the plant floor structure (Figure 2a). Since there was not any floor structure above the antennas near the discharge end of the screen, the chains holding these antennas were attached to temporary support structure constructed from PVC piping (Figure 2b).

After completing the installation of the antenna and electronics, two series of fullscale test runs were conducted to assess the automated system for detecting and counting the transponder-based tracers. The plant operating conditions for the test runs are summarized in Table 1. For first full-scale test, the antennas were mounted 12 inches off the surface of the screen deck and 7 inches from the screen edge. The tracer blocks were added into the process stream at a point where the feed to the fine heavy medium cyclone circuit comes off the upstream deslime

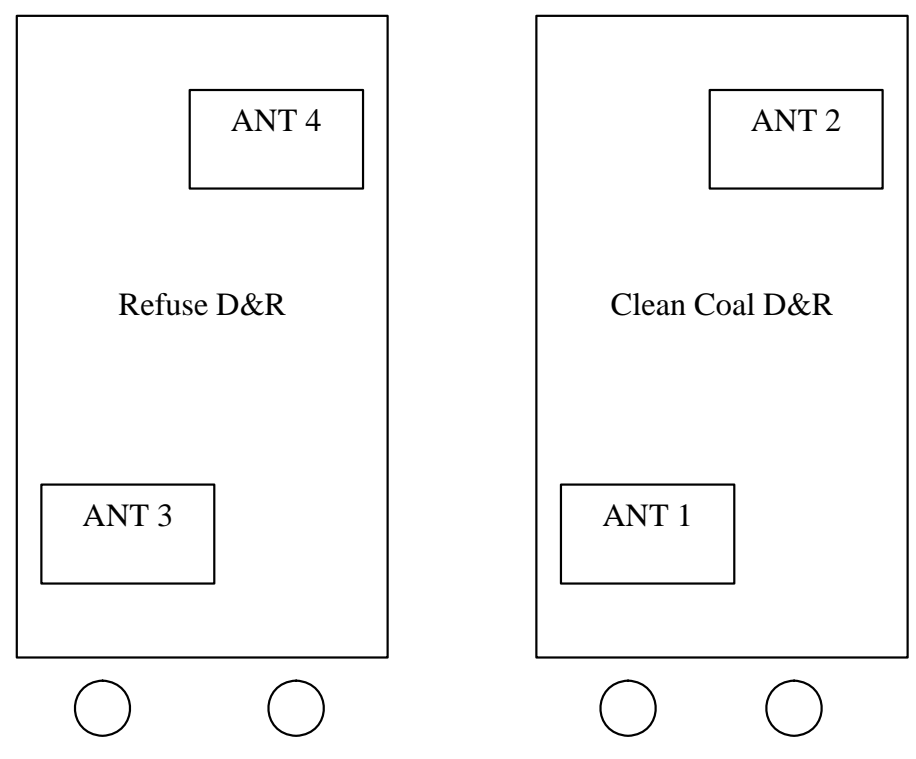

Figure 1. Antenna layout above the plant D\&R screens (circles show location of personnel). 

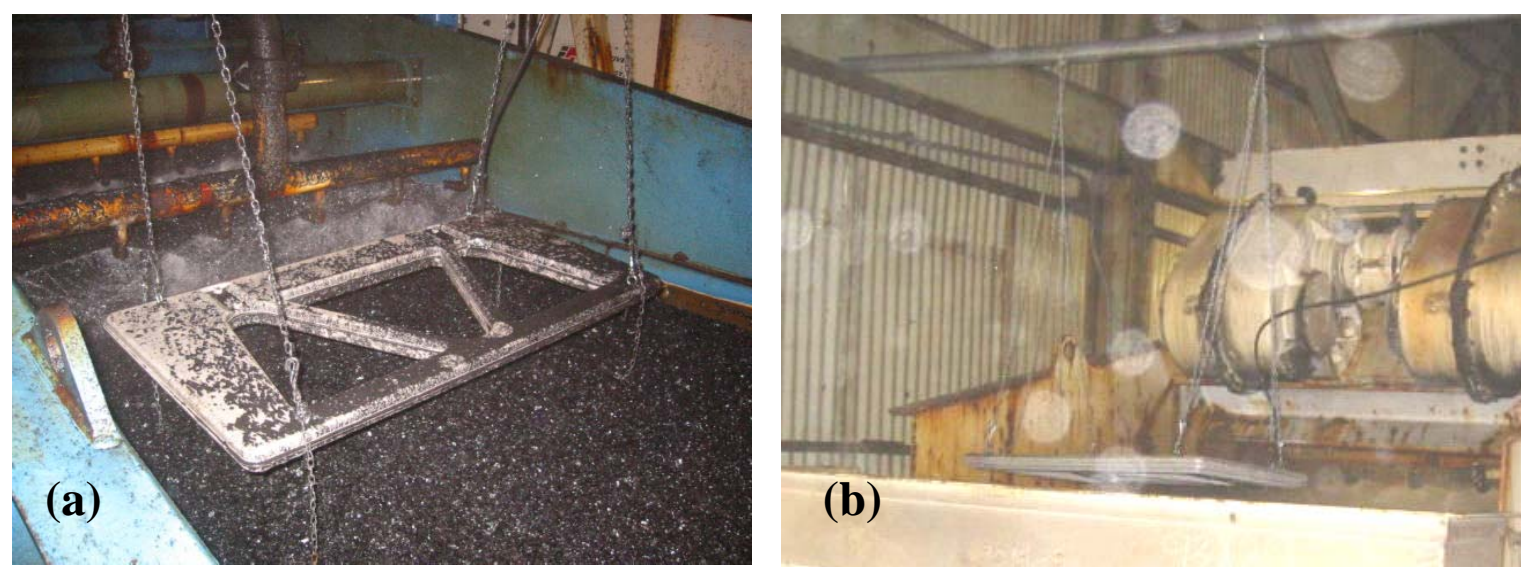

Figure 2. Antenna mounting to (a) plant structure and (b) temporary PVC piping.

screens. The tracers were manually introduced by hand into the feed stream at 5 second intervals. A hand-held antenna was used to electronically log the exact time that each tracer block added. For verification of the detection system, the tracer blocks were manually recovered by two persons stationed at the discharge end of each screen (see Figure 1).

A total of 378 density tracers with densities ranging from 1.3 to 1.9 SG were added in the first test run. In this case, $95 \%$ of the tracers added to the feed stream were collected by hand. The high recovery rate of tracers by hand was probably due to the large number of workers used to collect the tracers (i.e., two persons were stationed at each $6 \mathrm{ft}$ wide screen). Unfortunately, the electronic system detected only $75 \%$ of the blocks added to the feed stream. The low detection of the electronic tracer blocks was believed to be related to two separate issues. The first issue involved the location of the antennas. Diagnostic tests indicated that tracer blocks which passed near the very corners of the screens may have been missed by the antenna. This problem was corrected by relocating the antenna so that the end of each antenna was directly over the edge of each screen. The antennas were also lowered 3 inches to a distance of 9 inches

Table 1. Plant conditions for the tracer test runs.

\begin{tabular}{lcc}
\hline & Run \#1 & Run \#2 \\
\hline & 571 & 547 \\
Plant Feed Rate (tph) & 142 & 213 \\
Plant Clean Coal Rate (tph) & & \\
& 1.55 & 1.55 \\
Fine HMC SG PLC Setpoint & 1.65 & 1.66 \\
Fine HMC SG Cup Reading & 1.55 & 1.56 \\
Coarse HMC SG PLC Setpoint & 1.60 & 1.60 \\
Coarse HMC SG Cup Reading & & \\
& 19 & 19 \\
Fine HMC pressure (psi) & 30 & 30 \\
Diameter (inches) & 12 & 12 \\
Vortex finder (inches) & 9 & 9 \\
Apex (inches) & & \\
& 4 & 4 \\
Number of Antenna & 12 & 9 \\
Antenna (Inch Above Deck) & 3 & 0 \\
Antenna (Inch From Edge) & 5 & 15 \\
Tracer Addition Interval (sec) & & \\
&
\end{tabular}


above the screen deck to further improve the readability. The second issue involved a problem of tracer coincidence. Diagnostic tests showed that only one identification number was read when two transponders passed under an antenna at the same point in time. To eliminate this problem, the length of time between the addition of one tracer block and another was increased from 5 to 15 seconds.

A second test run was conducted using the new antenna positions and longer time spacing between tracer block additions. In this case, the number of tracer blocks added to the feed stream was substantially reduced from 378 to 65 . The reduction was considered reasonable to be necessary in order to minimize the losses of tracer blocks collected by hand. The test data from the second test run is provided in Table 2. As shown, 64 of 65 blocks (98.5\%) were detected and counted by the electronic system during the second test run. In comparison, 60 of 65 (92.3\%) blocks were collected manually by hand in the same test run. The electronic system was superior to the manual counting method despite the fact that two workers were placed at each $6 \mathrm{ft}$ screen in an attempt to ensure that the maximum possible blocks were collected by hand. The very high detection rate for the electronic system indicates that the modifications to the antenna location and changes to the timing of block additions greatly improved the system reliability.

Figure 3 shows the partition curve obtained using the density tracers. For comparison, the data are plotted for both the manual counting (hand collection) and electronic counting (transponder identification) methods. The Whiten formula was used to provide a best-fit regression curve through the data points obtained using the electronic tracers. The fitted data show that the plant heavy medium circuit was operating at a specific gravity cutpoint $\left(\mathrm{SG}_{50}\right)$ of 1.67 SG. The sharpness of the separation, as measured by the Ecart probable error (Ep),

Table 2. Data obtained from manual and electronic counting of density tracers.

\begin{tabular}{cccccccccccc}
\hline \multirow{2}{*}{ SG } & \multirow{2}{*}{ Feed } & \multicolumn{3}{c}{ Clean } & \multicolumn{3}{c}{ Refuse } & \multicolumn{3}{c}{ Total } & \multicolumn{3}{c}{ Collected (\%) } & \multicolumn{2}{c}{ Partition } \\
\cline { 2 - 12 } & Hand & EDT & Hand & EDT & Hand & EDT & Hand & EDT & Hand & EDT \\
\hline 1.30 & 5 & 4 & 5 & 0 & 0 & 4 & 5 & 80 & 100 & 0.00 & 0.00 \\
1.56 & 5 & 5 & 5 & 0 & 0 & 5 & 5 & 100 & 100 & 0.00 & 0.00 \\
1.58 & 5 & 4 & 5 & 0 & 0 & 4 & 5 & 80 & 100 & 0.00 & 0.00 \\
1.60 & 5 & 5 & 5 & 0 & 0 & 5 & 5 & 100 & 100 & 0.00 & 0.00 \\
1.62 & 5 & 4 & 4 & 0 & 0 & 4 & 4 & 80 & 80 & 0.00 & 0.00 \\
1.64 & 5 & 4 & 5 & 0 & 0 & 4 & 5 & 80 & 100 & 0.00 & 0.00 \\
1.66 & 5 & 4 & 4 & 1 & 1 & 5 & 5 & 100 & 100 & 0.20 & 0.20 \\
1.68 & 5 & 1 & 1 & 4 & 4 & 5 & 5 & 100 & 100 & 0.80 & 0.80 \\
1.70 & 5 & 0 & 0 & 5 & 5 & 5 & 5 & 100 & 100 & 1.00 & 1.00 \\
1.72 & 5 & 0 & 0 & 5 & 5 & 5 & 5 & 100 & 100 & 1.00 & 1.00 \\
1.74 & 5 & 0 & 0 & 4 & 5 & 4 & 5 & 80 & 100 & 0.80 & 1.00 \\
1.76 & 5 & 0 & 0 & 5 & 5 & 5 & 5 & 100 & 100 & 1.00 & 1.00 \\
1.90 & 5 & 0 & 0 & 5 & 5 & 5 & 5 & 100 & 100 & 1.00 & 1.00 \\
\hline Sum & 65 & 31 & 34 & 29 & 30 & 60 & 64 & $\mathbf{9 2 . 3}$ & $\mathbf{9 8 . 5}$ & -- & -- \\
\hline
\end{tabular}

Hand - manual collection, EDT - electronic density tracer system 
was found to be 0.008 . This very good efficiency number, combined with the fact that the ends of the curve close at low and high SG values, indicated that no gross misplacement of either coal or rock occurred for this particular test run. More importantly, the automated system was able to numerically quantify these key indicators of circuit performance in just a matter of minutes using only a single person.

\section{SUMMARY}

Field tests were carried out to evaluate the capabilities of the proposed electronic detection system for counting density tracers. The test runs were performed by passing density tracers embedded with passive transponders through a heavy medium cyclone circuit at an industrial plant site. Several problems were encountered during the initial test run that resulted in poor detection levels for the electronic tracers. These problems, which included improper antenna heights and excessively rapid tracer introduction, were corrected by simple changes to the radio equipment and test protocols. After making these modifications, very accurate counts of the transponder-based tracers were achieved. In the final test run, 98.5\% of the electronically tagged tracer blocks were detected by the automated counting system. This level of detection was superior to the $92.3 \%$ collection rate achieved using manual (hand) collection. The superior performance was particularly impressive considering that two employees were used per $6 \mathrm{ft}$ width of screen to ensure that very high manual collection rates were maintained. The manual collection rate is normally much lower in actual practice since (i) fewer personnel are normally available for collecting the density tracers and (ii) few plant sites are as ideally suited for block collection as the one employed for this test work.

\section{FUTURE WORK}

Work conducted during the next reporting period will focus on further refinement of the user interfaces for the electronic density tracer system. In addition, a cost analysis will be performed to assess the overall cost of installing and operating the prototype system at an industrial plant site. Work will also begin on the preparation of a final technical report for this research project. 


\section{REFERENCES}

1. DASYLab, “Book 1: User Guide” and "Book 2 Module Reference Guide,” DASYTECH USA, A National Instruments Company, NH, 1991-2001.

2. Scher, Bob, “Antenna Considerations for Low Frequency RFID Applications," http://www.rfidusa.com/rfid_antenna_consid.html.

3. Sorrells, Peter, 2000 "Optimizing Read Range in RFID Systems," EDN Magazine, December 7, 2000, pp. 173-184.

4. Texas Instruments, Series 2000 Reader System, RI-RFM-007B, Reference Guide

5. WinWedge, “RS-232 User Guide,” TAL Technologies, PA, www.taltech.com.

\section{PUBLICATIONS/PRESENTATIONS}

To date, no major publications have resulted from this project. 